\title{
Photonic-crystal slow-light enhancement of nonlinear phase sensitivity
}

\author{
Marin Soljačić and Steven G. Johnson \\ Department of Physics and Center for Materials Science and Engineering, Massachusetts Institute of Technology, \\ Cambridge, Massachusetts 02139 \\ Shanhui Fan \\ Department of Electrical Engineering, Stanford University, Stanford, California 94305
}

Mihai Ibanescu, Erich Ippen, and J. D. Joannopoulos

Department of Physics and Center for Materials Science and Engineering, Massachusetts Institute of Technology, Cambridge, Massachusetts 02139

\begin{abstract}
Received November 15, 2001; revised manuscript received February 15, 2002; accepted February 21, 2002
We demonstrate how slow group velocities of light, which are readily achievable in photonic-crystal systems, can dramatically increase the induced phase shifts caused by small changes in the index of refraction. Such increased phase sensitivity may be used to decrease the sizes of many devices, including switches, routers, all-optical logical gates, wavelength converters, and others. At the same time a low group velocity greatly decreases the power requirements needed to operate these devices. We show how these advantages can be used to design switches smaller than $20 \mu \mathrm{m} \times 200 \mu \mathrm{m}$ in size by using readily available materials and at modest levels of power. With this approach, one could have $\sim 10^{5}$ such devices on a surface that is $2 \mathrm{~cm}$ $\times 2 \mathrm{~cm}$, making it an important step towards large-scale all-optical integration. (C) 2002 Optical Society of America

OCIS code: 900.9040
\end{abstract}

\section{INTRODUCTION}

The size of high-speed active elements is currently a critical problem in the path toward large-scale optical integration. The smallest all-optical and electro-optical switches are of the order of millimeters ${ }^{1-3}$ with little promise of getting much smaller. The reason for this problem is that the changes in the index of refraction induced by electro-optical or nonlinear optical effects, which are used to operate the devices, are very small [ $\delta n$ is $\mathcal{O}(0.001)]$. If one wants to use an induced $\delta n$ to shift the phase of a signal by $\pi$ after propagating through a length $L$ of some material, the induced phase change is then $\pi$ $=2 \pi L \delta n / \lambda_{\mathrm{AIR}} \Rightarrow L=\lambda_{\mathrm{AIR}} / 2 \delta n$, requiring the size of the device to be millimeters or more. (A phase change of $\pi$ can be used to switch the signal on or off by one's placing the material in an interferometer. For example, one could use a Mach-Zehnder interferometer, which we describe below.)

It is well known that nonlinear effects can be enhanced in systems with slow group velocity as a result of the compression of the local energy density. Our observation in this paper is that the sensitivity of the phase to the induced change in the index of refraction can be drastically enhanced if one operates in the regimes of slow group velocities. Slow group velocities occur quite commonly in photonic crystals and in systems with electromagnetically induced transparency. ${ }^{4-7}$ According to perturbation theory, the induced shift in the optical frequency of a pho- tonic band mode at a fixed $k$ that is due to a small $\delta n$ is given by $\delta \omega(k) / \omega=-\sigma(\delta n / n)$ where $\sigma$ specifies the fraction of the total energy of the mode in question that is stored in the region where $\delta n$ is being applied. However, because the induced phase shift actually depends on $\delta k$, the phase shift can be greatly enhanced if $v_{G}$ $=\mathrm{d} \omega / \mathrm{d} k$ is small, as illustrated in Fig. 1. More precisely, the induced phase shift is $\delta \phi=L * \delta k$ $\approx L * \delta \omega /(\mathrm{d} \omega / \mathrm{d} k) \Rightarrow \delta \phi \approx L \omega \sigma \delta n /\left(n v_{G}\right)$. In other words, if $\delta \phi=-\pi$, we have

$$
\frac{L}{\lambda_{\mathrm{AIR}}} \approx \frac{1}{2 \sigma}\left(\frac{n}{\delta n}\right)\left(\frac{v_{G}}{c}\right),
$$

or for a given $\delta n$ the size of the device scales linearly with $v_{G}$.

An electro-optical device that is smaller in length by a factor of $v_{G} / c$ also requires $v_{G} / c$ less power to operate, which is perhaps an even more important consideration for large-scale integration. Thus for an electro-optic modulator or a switch the device enhancement is a factor of $\left(v_{G} / c\right)^{2}$. The same improvement by $\left(v_{G} / c\right)^{2}$ is achieved in an all-optical gate by use of the Kerr effect. In this case, the $\delta n$ change is self-induced by the signal itself, and $\delta n$ is proportional to the local electric field squared. The savings in the length of the device is the same $v_{G} / c$ as for an electro-optic device. In addition, because of the small $v_{G}$, the energy of the pulse is temporally compressed by a factor of $v_{G} / c$, so the induced $\delta n$ is 


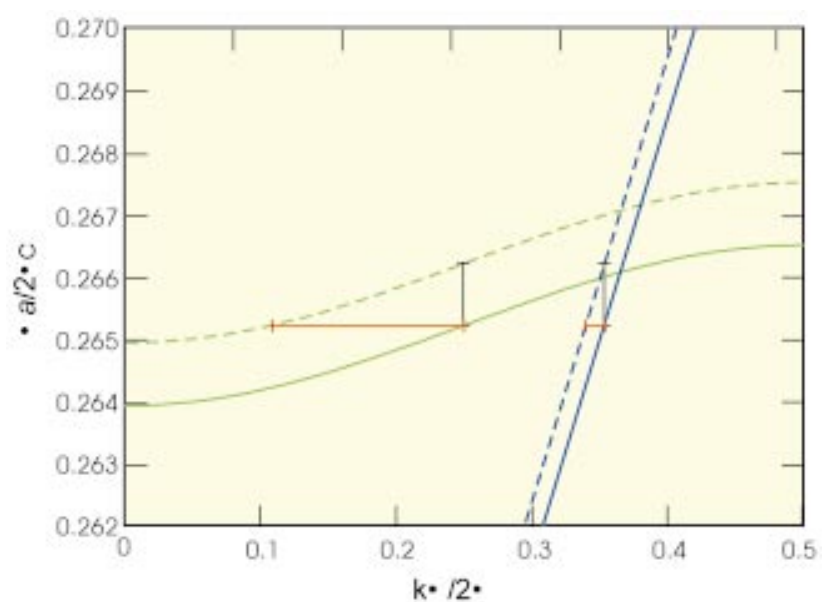

Fig. 1. Induced change in the photonic band frequency in a material depends mostly on the induced index of refraction change. However, depending on the local group velocity, this can lead to drastically different changes in the wave vector. Here we show this effect for two dispersion curves: the slow-light band with $v_{G}=0.022 c$ used in a device proposed in this paper (green), and the dispersion curve of a uniform material with $n=3.5$ (blue). We apply the same frequency shift $(\delta \omega=0.001)$ to both dispersion curves to get the respective dashed curves. As we can see, the same $\delta \omega$ (black) leads to two very different $\delta k$ (red).
$1 /\left(v_{G} / c\right)$ times larger. Thus for a pulse of a fixed total energy, we can actually use a device that is smaller by a factor of approximately $\left(v_{G} / c\right)^{2}$.

\section{IMPROVEMENTS OFFERED BY PHOTONIC CRYSTALS}

Photonic crystals (PCs) are ideal systems in which one can achieve arbitrarily low group velocities. ${ }^{8-10}$ PCs are artificially created materials in which the index of refraction has a one-dimensional, a two-dimensional (2-D), or a three-dimensional (3-D) periodicity. Under appropriate conditions and when the maximum index contrast is sufficiently large, a photonic bandgap appears: a range of frequencies in which light cannot propagate in the crystal. Because of this gap, photons inside a PC have many properties that are similar to electrons in semiconductors. Consequently, PCs are considered to be promising media for large-scale integrated optics. In particular, line defects in a PC can lead to guided-mode bands inside the photonic bandgap. These bands can, in principle, be made as flat as desired by the appropriate design. Typical group velocities for reasonable linear defect guided modes can easily be $\mathcal{O}\left(10^{-2} c-10^{-3} c\right)$, thus making it possible to shrink the size of all-optical and electro-optical

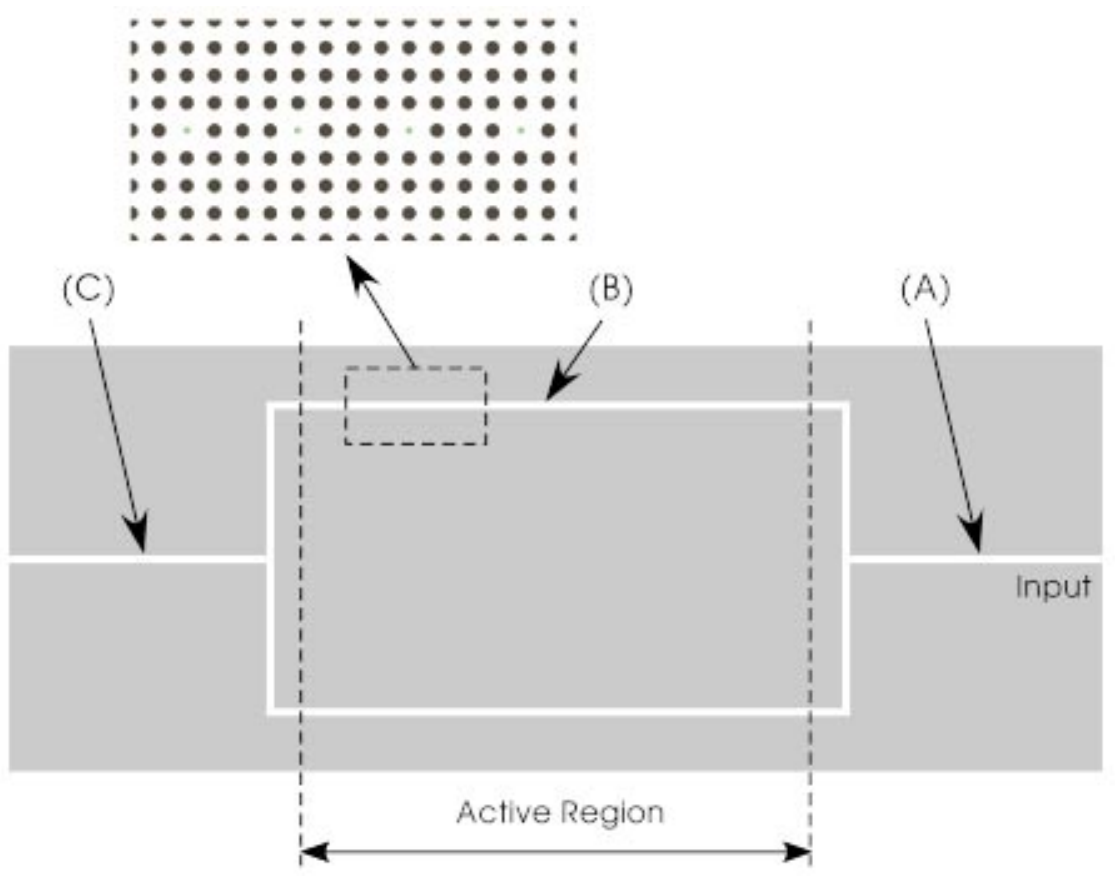

Fig. 2. Sketch of the Mach-Zehnder interferometer that we used to demonstrate enhancement of nonlinear phase sensitivity that is due to slow light in PCs. The slow-light system that we use is a coupled-cavity waveguide (CCW), as shown in the upper left-hand enlarged area of this figure. (Throughout the paper, cavities are colored green to make them more visible). The signal enters the device on the right, is split equally at the first $\mathrm{T}$ branch into the upper and the lower waveguides, and recombines at the $\mathrm{T}$ branch on the left. If no index change is induced, the parts of the signal coming from the top and the bottom interfere constructively at the second T branch, and the pulse exists entirely at the output. If we induce the index change in the active region in an appropriate manner, the two parts of the signal interfere destructively, and the pulse is reflected back toward the input, with no signal being observed at the output. The points marked (A), (B), and (C) correspond to field-monitor points during the simulations. The results of observations from these points are displayed in Fig. 3. 
devices in PCs by approximately $\left(v_{G} / c\right)^{2} \sim 10^{-4}-10^{-6}$, while keeping the operating power fixed. Group velocity of $c / 100$ was experimentally demonstrated in a photonic crystal system recently. ${ }^{11}$

For definiteness, we focus our attention on a particular class of PC line-defect systems that can have low group velocities; namely, we discuss coupled-cavity waveguides $^{12,13}$ (CCWs). Nonlinear properties of CCWs (also called coupled resonator optical waveguides) were very recently summarized in Ref. 14. A CCW consists of many cavities, as shown in Fig. 2. Each of these cavities when isolated supports a resonant mode with the resonant frequency well inside the bandgap. When we bring such cavities close to each other to form a linear defect, as is shown in Fig. 2, the photons can propagate down the defect by tunneling from one cavity to another. Consequently, the group velocity is small, and the less closely coupled the cavities are, the slower the group velocity. Group velocities of $c / 1000$ or even smaller are easy to attain in such systems.

Because the cavities in a CCW are so weakly coupled to each other, the tight-binding method ${ }^{12-17}$ is an excellent approximation in deriving the dispersion relation. The result is $\omega(k)=\Omega[1-\Delta \alpha+\kappa \cos (k \Lambda)]$, where $\Omega$ is the single-cavity resonant frequency, $\Lambda$ is the physical distance between the cavities, and $\Delta \alpha \ll \kappa \ll 1$ in the tightbinding approximation, so we can approximate $\Delta \alpha=0$ in our analysis.

The CCW system has a zero-dispersion point at $k=\pi / 2 \Lambda$. We choose this to be the operation point of our devices because the devices in that case have far larger bandwidths than most other slow-light systems would have; in our simulations the useful bandwidth of such CCW devices is typically more than $1 / 3$ of the entire CCW band. (For example, in a Mach-Zehnder interferometer, useful bandwidth would be defined as the range of values of $\omega$ in which the extinction ratio is less than $99 \%$ when the device is in its OFF state.) To see that the CCW system is optimal with respect to bandwidth, we note that, to maximize the bandwidth, one wants $\Delta k$ $\equiv k(\omega, n+\delta n)-k(\omega, n)$ to be as independent of $\omega$ as possible, for as large a range of $\omega$ as possible. One way to satisfy this condition is, for example, if $k(\omega)$ is nearly linear and if the dominant effect of $\delta n$ is to shift $k(\omega)$ upward or downward by a nearly constant amount. This approach is precisely what happens in CCW systems. First, note that $\omega(k, \delta n)=\Omega\left(1+\delta_{1}\right)[1+\kappa(1$ $\left.+\delta_{2}\right) \cos (k \Lambda)$ ], where $\delta_{1}$ and $\delta_{2}$ are the first order in $\delta n$. Thus $\delta n$ shifts the curve upward by $\Omega \delta_{1}$ and also changes the slope at the zero-dispersion point: $\kappa \rightarrow \kappa\left(1+\delta_{1}\right)(1$ $+\delta_{2}$ ). But, because the slope was so small to start with (as $\kappa \ll 1$ in slow-light systems), the dominant effect in $\Delta k(\omega, \delta n)$ is the linear shift upward, which produces a term independent of $\omega$.

To make the claim from the previous paragraph more precise, we can expand $\omega(k, \delta n)$ about the zerodispersion point and invert the relation to get $k(\omega, \delta n)$ and thereby $\Delta k(\omega, \delta n) \approx\left[\delta_{1} \omega / \Omega+\delta_{2}(\omega / \Omega-1)\right] / \Lambda \kappa$. Moreover, because we are working with a slow-light band, we are interested only in $\omega$ that can be written as $\omega=\Omega+\delta \omega$, where $\delta \omega \ll \Omega$. Thus $\Delta k(\delta \omega, \delta n) \approx\left\{\delta_{1}\right.$ $\left.+\left[(\delta \omega / \Omega) *\left(\delta_{1}+\delta_{2}\right)\right]\right\} / \Lambda \kappa$, and, because $\delta \omega / \Omega \ll 1, \Delta k$ is almost constant across most of the slow-light band. In fact, a similar derivation can easily be adapted to apply for any zero-dispersion point of any flat dispersion curve. Consequently, if one wants to use slow light to enhance the nonlinear phase sensitivity, one should operate at a zero-dispersion point because this optimizes the available bandwidth of the device, even in non-CCW systems.

\section{SLOW-LIGHT PHOTONIC-CRYSTAL MACH-ZEHNDER DEVICES}

To demonstrate how the ideas presented above can be put to work in practice, we perform numerical-simulation studies of a 2-D CCW system. The system is illustrated in Fig. 2. It consists of a square lattice of high- $\epsilon$ dielectric rods $\left(\epsilon_{H}=12.25\right)$ embedded in a low- $\epsilon$ dielectric material $\left(\epsilon_{L}=2.25\right)$. The lattice spacing is denoted $a$, and the radius of each rod is $r=0.25 a$. The CCW is created by the reduction of the radius of each fourth rod in a line to $r / 3$, so $\Lambda=4 a$. We focus our attention on TM modes, which have electric fields parallel to the rods.

To begin our study of the modes of this system, we perform frequency-domain calculations by using preconditioned conjugate gradient minimization of the Rayleigh quotient in a 2-D plane-wave basis, as described in detail in Ref. 18. To model our system, we employ a supercell geometry of size $(11 a \times 4 a)$ and a grid of 72 points $/ a$. The results reveal an $18 \%$ photonic bandgap between $\omega_{\mathrm{MIN}}=0.24(2 \pi c) / a$ and $\omega_{\mathrm{MAX}}=0.29(2 \pi c) / a$. Adding a line defect of CCWs mutually spaced $\Lambda$ apart leads to a CCW band that can be excellently approximated by $\omega=[0.26522-0.00277 \cos (k \Lambda)](2 \pi c / a)$. This gives $v_{G}$ $=0.0695 \mathrm{c}$ at the point of zero dispersion for the case of $\Lambda=4 a$. (To demonstrate our ideas, we use a $v_{G}$ that is not extraordinarily small to be less demanding on the time-domain numerical simulations.) The frequencydomain calculation further tells us that roughly $50 \%$ of the energy of each mode is in the high- $\epsilon$ regions and that this ratio is fairly independent of $k$.

To put things in perspective, we can use the frequencydomain code results to estimate sizes of some real devices. For purposes of illustration only, we simulate a device in which we modify the high- $\epsilon$ material by $\delta n / n_{H}=2$ $\times 10^{-3}$ to perform the switching, say, through an electrooptical effect. We pick a somewhat unrealistically large $\delta n / n_{H}$ to lower the requirements on our numerical simulations; as mentioned earlier, the length of the system varies inversely with $\delta n / n_{H}$, and we can scale our results to experimentally realizable $\delta n$ accordingly. Let us now ask how long the CCW has to be for the signal to accumulate an exact $\pi$ phase shift when propagating down this $\mathrm{CCW}$ compared with the case when $\delta n=0$. We run the frequency-domain code twice with the two different values of $\epsilon_{H}$ for 18 uniformly distributed $k$ between $k=0$ and $k$ at the edge of the first Brillouin zone. Next, we perform the tight-binding curve fits to the two sets of data. From these two fitted curves it is trivial to get $\Delta k$ at the point of zero dispersion and thereby $L$ from the relation $L \Delta k=\pi$. The result is that the CCW has to be approximately $L=31.6 \Lambda=35.5 \lambda_{\mathrm{AIR}}$ long to achieve a $\pi$ phase shift when propagating down this CCW. 
Switch-ON
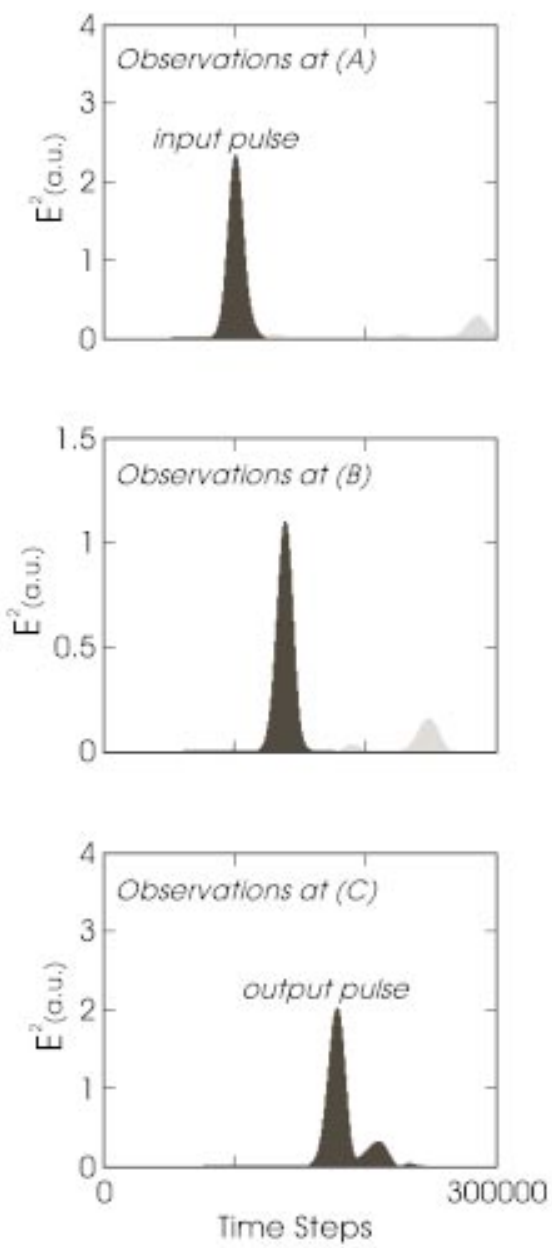

Switch-OFF
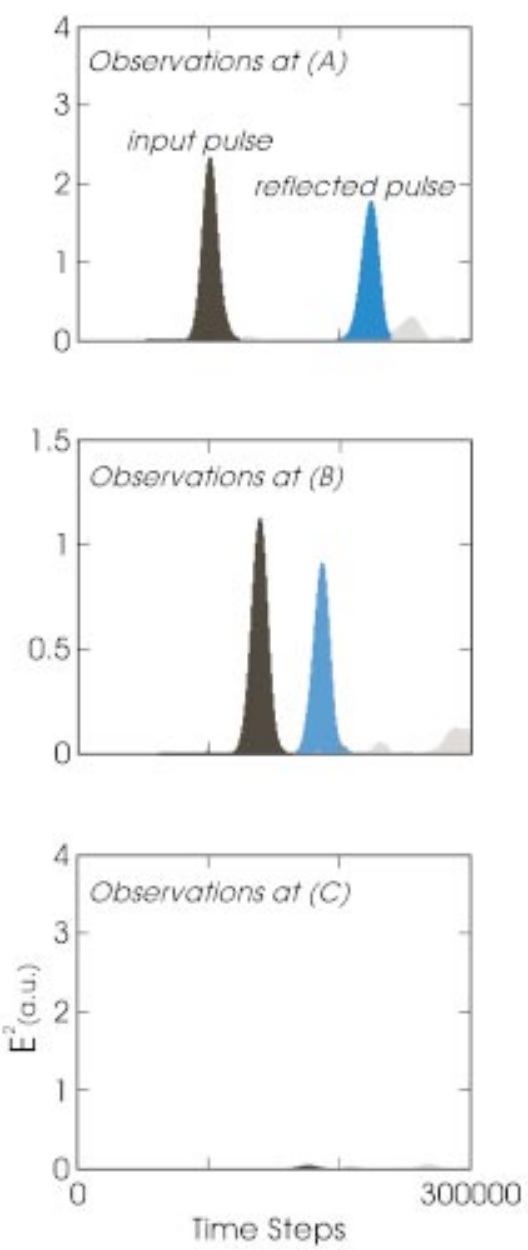

Fig. 3. Demonstration of nonlinear switching in a slow-light PC system. The electric field squared is plotted as a function of time, observed at three different points: (A), (B), and (C) in the system of Fig. 2. The left-hand column corresponds to the case when no index change is induced; most of the incoming signal at (A) exits at the output (C). The column on the right-hand side corresponds to the case when the nonlinear index change is induced; the signal at the output $(\mathrm{C})$ in the right-hand column is drastically reduced compared with the output (C) in the left-hand column. The black and the blue signals represent the pulses traveling from right to left and left to right, respectively, in Fig. 2. The gray pulses are spurious reflections (mostly from the interface between the PC and air at the exits of the device).

Based on what we learned from the frequency-domain calculations, we can now design a Mach-Zehnder interferometer for use in the time domain. Specifically, we perform 2-D finite-difference time-domain simulations ${ }^{19}$ with perfectly matched layer absorbing boundary conditions. ${ }^{20}$ Our numerical resolution is $24 \times 24$ points $/ a^{2}$, meaning that the entire computational cell is $5000 \times 696$ points. Because our computational cell is large enough, we can easily distinguish the pulses reflected from the boundaries of our simulation from the real physical pulses. For the waveguides, we use the CCWs as described above. The time-domain simulation reveals that there is a slow-light band between $\omega_{\mathrm{MIN}}=0.2620(2 \pi c) / a$ and $\omega_{\mathrm{MAX}}=0.2676(2 \pi c) /$ $a$, which is within $0.2 \%$ of the frequency-domain prediction. Next, we use results of previous research to implement the needed $90^{\circ}$ bends $^{21}$ and $\mathrm{T}$ branches. ${ }^{22}$ At the input of our Mach-Zehnder interferometer, we launch a pulse with a Gaussian frequency profile, a carrier frequency of $\omega_{0}=0.2648(2 \pi c) / a$, and a FWHM of $\Delta \omega / \omega_{0}=1 / 200$. During the simulations, we monitor the electric field $E(t)$ at eight points in the system. We show the placement of three of these points in Fig. 2. The observations at the other five points (which include the branch points and the end points) provide us with no new information but are monitored just to ensure selfconsistency. When $\delta n$ is OFF, as in the first column of Fig. 3, the signal comes in at the input, splits equally into the two arms at the first branch, and recombines at the second branch, traveling toward the output, as seen in Fig. 4. Apart from very small reflections (approximately $2 \%$ ) that are due to the lack of full, optimized branches, most of the signal reaches the output. Next we change the high-index material to $n_{H} \rightarrow(1+0.00166) n_{H}$ in the upper half of the active region shown in Fig. 2 and $n_{H} \rightarrow(1-0.00166) n_{H}$ in the lower half of the active region shown in Fig. 2. ${ }^{23}$ Because of this difference in $n_{H}$, the part of the signal traveling in the upper arm accumu- 
lates $\pi$ more phase shift than the half traveling in the lower arm. ${ }^{24}$ Thus the signal interferes destructively at the output, and is reflected back toward the input, as can be seen from Fig. 5. Even without any fine tuning, we observe a signal at the output that is $16.4 \mathrm{~dB}$ smaller than in the case when $\delta n$ is not applied.

As was mentioned above, CCWs have an added benefit in that the bandwidth of the device above is optimal because of the existence of the zero-dispersion point. For example, the useful bandwidth of the device of Figs. 2-5 is $\Delta \omega / \omega_{0} \approx 1 / 150$. The useful bandwidth of the CCW devices scales roughly linearly with $v_{G} / \Lambda$ for small $\delta n$. On the other hand, the performance gets better with $1 / v_{G}^{2}$. Therefore, if we are willing to have a device with a smaller useful bandwidth, we can have a much more efficient device. For example, a 40-Gbit/s telecommunications stream has a bandwidth of $\Delta \omega / \omega_{0} \approx 1 / 3000$, so we can afford to operate the device with pulses that have 400 times less energy than when using the device of Figs. 2-5 for the same device size. An additional savings of power occurs in CCW systems because most of the power is tightly confined to the cavities. In contrast, in a uniform waveguide power is uniformly distributed along it. This fact typically produces an additional power savings of a factor of 2-3 or more, depending on the geometry of the system.

To make the idea of the previous paragraph a bit more explicit, we explore the possibility of an all-optical device with more optimized parameters. We envision a system similar to that of Figs. 2-5 but with coupled cavities spaced six lattice periods apart, so $\Lambda=6 a$. In this case the group velocity is $0.022 c$ (approximately a factor of 3 slower than in the case above). Now we use the distribution of the fields given by the frequency-domain code to calculate [by using first-order perturbation theory in the small quantity $\delta n(\mathbf{r}) / n(\mathbf{r})]$ what happens to the slowlight band after the nonlinear Kerr effect is turned on. We apply the Kerr effect only in the high-index regions because high-index materials typically have nonlinear coefficients that are much higher than those of low-index materials. We now pick physically realistic parameters so that the largest induced $\delta n / n$ anywhere is $2 \times 10^{-4}$. In this case, the perturbation theory tells us that, if we operate at the zero-dispersion point and the Kerr effect is present only in one arm of the interferometer, the length of the device has to be $70.97 \Lambda=175 \mu \mathrm{m}$ at $\lambda=1.55 \mu \mathrm{m}$. The transverse size of the device would then be roughly $20 \mu \mathrm{m}$. Suppose that our high-index material has a Kerr coefficient of $n_{2}=1.5 \times 10^{-13} \mathrm{~cm}^{2} / \mathrm{W}$ (which is a value achievable in a number of materials, GaAs at $\lambda_{0}=1.55 \mu \mathrm{m}$ being one of them), where the Kerr effect is given by $\delta n=n_{2} I$. In that case, we would need roughly $0.26 \mathrm{~W}$ of peak power to operate our device. (For comparison, an integrated-optics device made of same (uniform) high-index material with a cross-sectional area of $0.5 \mu \mathrm{m} \times 0.5 \mu \mathrm{m}$ would have to be $5 \mathrm{~cm}$ long to operate at the same 0.26 -W peak power.) The useful bandwidth of our device would be at least $\Delta \omega / \omega_{0} \approx 1 / 670$, meaning that it could operate at bit rates greater than $100 \mathrm{Gbits} / \mathrm{s}$. For lower bit rates with less bandwidth the performance
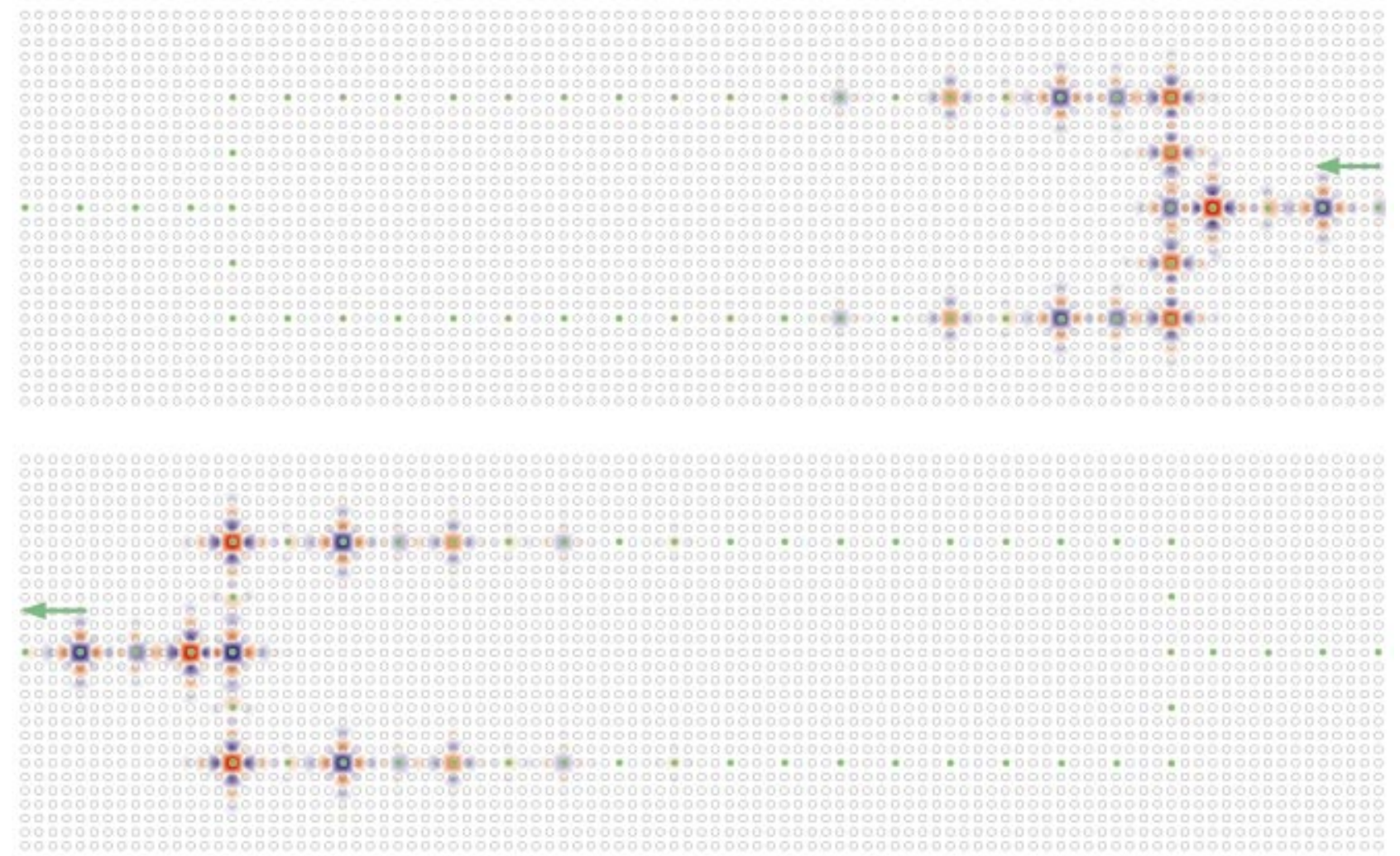

Fig. 4. Snapshots of the electric field pulse in the system of Fig. 2 for the case when no index change is induced. The top panel represents 120,000 time steps, and bottom panel 160,000 time steps. The signal entering at the input exits at the output of the device; the device is in its ON state. 

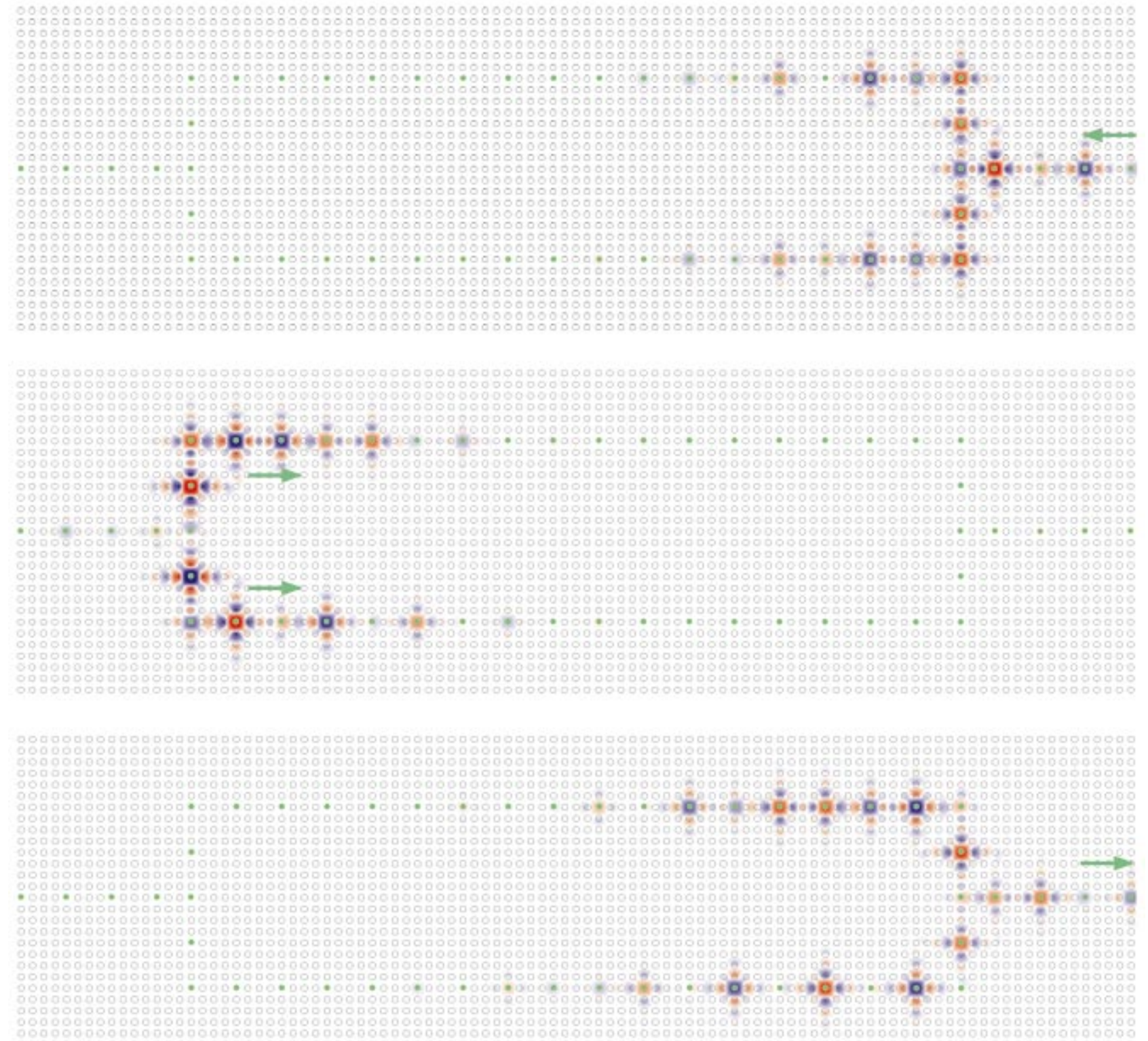

Fig. 5. Snapshots of the electric field pulse in the system of Fig. 2 for the case when an index change is induced. Top panel represents 120,000 time steps, the middle panel 160,000 time steps, and the bottom panel 200,000 time steps. The signal entering at the input is reflected back toward the input; device is in its OFF state.

enhancements could be even greater. Finally, even the design outlined here can be further improved by our confining more energy into high- $\epsilon$ regions, (for example, by use of a triangular-lattice PC system with air holes in dielectric) or by our increasing the index contrast between the high- $\epsilon$ and the low- $\epsilon$ materials. By optimizing the design along these lines, one should be able to enhance the system by additional factors of 3-4 without much effort.

\section{PHOTONIC CRYSTAL $1 \times 2$ SWITCH}

The switching mechanism in this paper is general enough for use in a variety of all-optical logical operations, switching, routing, wavelength conversion, and optical imprinting. For example, we can enhance the applicability of our design by allowing the device to have two out puts (as shown in the upper plot of Fig. 6) instead of just one (as shown in Fig. 2). In this case the nonlinear mechanism in question directs input to either of the two outputs. To achieve this, we put a directional coupler at the output of the device instead of terminating the device with a simple branch. The directional coupler has to be designed so that (depending on the relative phase of its two inputs) it directs both of them either to the upper or the lower of its two outputs. Almost any directional coupler can be designed to perform this function.

A PC implementation could be based on the waveguide drop-filter design of Ref. 25. For this application, we need only to operate it at a frequency that is offset from that originally intended. An advantage of this particular design is that it adds only $1 \lambda$ to the length of the entire device. The device of Ref. 25 involves two linear 
waveguides and a coupling element (consisting of two point-defect cavities) between them, as shown in the inset of the upper panel of Fig. 6. If we label the waveguides as 1 and 2 , we can write

$$
\begin{array}{r}
A_{\mathrm{OUT} 1}=A_{\mathrm{IN} 1}\left(1-\frac{i \alpha}{\omega-\omega_{0}+i \alpha}\right) \\
-A_{\mathrm{IN} 2}\left(\frac{i \alpha}{\omega-\omega_{0}+i \alpha}\right), \\
A_{\mathrm{OUT} 2}=A_{\mathrm{IN} 2}\left(1-\frac{i \alpha}{\omega-\omega_{0}+i \alpha}\right) \\
-A_{\mathrm{IN} 1}\left(\frac{i \alpha}{\omega-\omega_{0}+i \alpha}\right),
\end{array}
$$

where $A_{\text {OUT } j}$ and $A_{\text {IN } j}$ are the amplitudes at the output and the input, respectively, of waveguide $j$ the central fre-
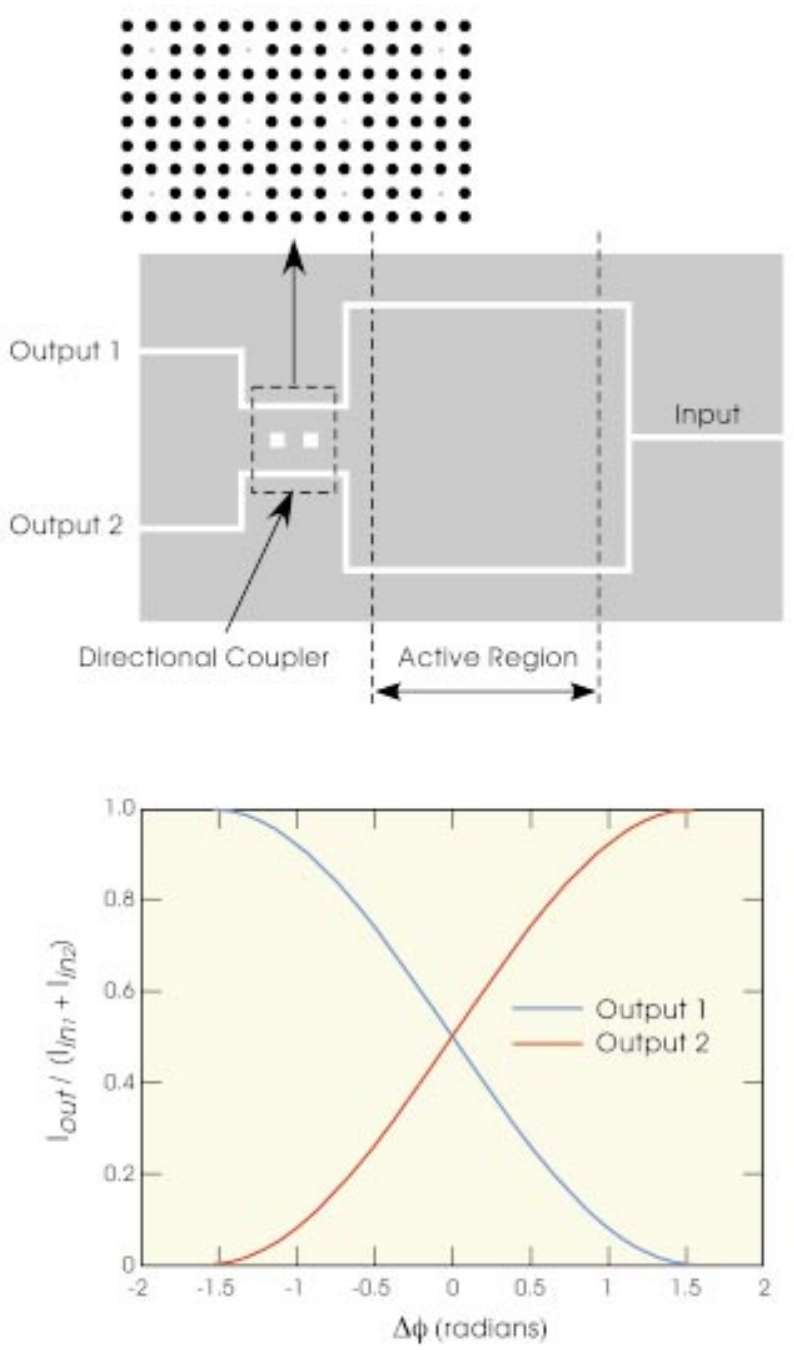

Fig. 6. Mach-Zehnder interferometer operating as a router between two different outputs. This operation is achieved by the termination of the interferometer with a directional coupler rather than with a $\mathrm{T}$ branch, as shown in the upper panel. The lower panel gives the calculated intensity at each of the two outputs as a function of the phase difference between the upper and the lower input to the directional coupler. quency of the two coupled cavities is $\omega_{0}$, and $2 \alpha$ is the width of the resonance. In our device, we will have equal intensities coming to both inputs, and we would like all the energy to exit at a single output. If we look at this picture from a time-reversed perspective, this tells us that we have to operate the directional coupler at the frequency $\omega_{0} \pm \alpha$, rather than operating it at $\omega=\omega_{0}$, as was done in Ref. 25. This time-reversed picture also tells us that [according to Eqs. (2)], if we choose to operate at frequency $\omega_{0}+\alpha$, we get $100 \%$ transmission at the output of waveguide 2 if the input of waveguide 2 lags waveguide 1 in phase by exactly $\pi / 2$. We get $100 \%$ transmission at the output of waveguide 1 if the input of waveguide 2 is $\pi / 2$ ahead. The dependence of transmission on the phase difference $\Delta \phi$ between waveguides 1 and 2 is illustrated in the lower plot of Fig. 6. Operating at the frequency $\omega_{0}-\alpha$ reverses this relative phase dependence.

For example, let us pick as the operating frequency $\omega_{0}+\alpha$ and say that the intensities entering the device from the two waveguides are the same distance apart for the fact that waveguide 2 lags waveguide 1 in phase by $\pi / 2$. Then

$$
\begin{gathered}
\frac{I_{\mathrm{OUT} 1}}{I_{\mathrm{IN} 1}+I_{\mathrm{IN} 2}}=\frac{1}{2} \frac{\left(\omega-\omega_{0}-\alpha\right)^{2}}{\left(\omega-\omega_{0}\right)^{2}+\alpha^{2}}, \\
\frac{I_{\mathrm{OUT} 2}}{I_{\mathrm{IN} 1}+I_{\mathrm{IN} 2}}=\frac{1}{2} \frac{\left(\omega-\omega_{0}+\alpha\right)^{2}}{\left(\omega-\omega_{0}\right)^{2}+\alpha^{2}},
\end{gathered}
$$

where $I_{\text {IN1,IN2 }}$ and $I_{\text {OUT1,OUT2 }}$ denote the intensities at the inputs and the outputs of the respective waveguides. According to Eqs. (3), the useful bandwidth of this directional coupler approximately equals $2 \alpha$. In contrast to Ref. 25, we are not forced to operate in the regime of very small $\alpha$; consequently, $2 \alpha$ can readily be designed to be larger than the bandwidth of our Mach-Zehnder interferometer, so the directional coupler will not impair the performance of the device.

\section{CONCLUDING REMARKS}

The sizes of the nonlinear devices described in this paper that use physically realistic values of $\delta n$ are small enough to contain $10^{5}$ of them on a chip of surface size $2 \mathrm{~cm}$ $\times 2 \mathrm{~cm}$, operated at moderate pulse energy levels, and with speeds greater than $100 \mathrm{Gbits} / \mathrm{s}$. Therefore, we view the research described here as an important step toward enabling large-scale integration of truly all-optical logic circuits. Finally, it should be emphasized that all the results and arguments presented in this paper (which has focused on simplified 2-D models) apply immediately to three dimensions. In fact, very recently, new 3-D PC structures have been introduced that reproduce the properties of linear and point defect modes in 2-D PC systems. ${ }^{26,27}$ Such 3-D structures should prove to be ideal candidates for the eventual practical realization of the nonlinear slow-light designs discussed in this paper. 


\section{ACKNOWLEDGMENT}

This study was supported in part by the Materials Research Science and Engineering Center program of the National Science Foundation under grant DMR9400334.

\section{REFERENCES AND NOTES}

1. W. E. Martin, "A new waveguide switch/modulator for integrated optics," Appl. Phys. Lett. 26, 562-564 (1975).

2. K. Kawano, S. Sekine, H. Takeuchi, M. Wada, M. Kohtoku, N. Yoshimoto, T. Ito, M. Yanagibashi, S. Kondo, and Y. Noguchi, " $4 \times 4$ InGaAlAs/InAlAs MQW directional coupler waveguide switch modules integrated with spot-size converters and their 10-Gbit/s operation," Electron. Lett. 31, 96-97 (1995).

3. A. Sneh, J. E. Zucker, and B. I. Miller, "Compact, low-crosstalk, and low-propagation-loss quantum-well Y-branch switches," IEEE Photonics Technol. Lett. 8, 1644-1646 (1996).

4. S. E. Harris, "Electromagnetically induced transparency," Phys. Today 50, 36-42 (1997).

5. L. V. Hau, S. E. Harris, Z. Dutton, and C. H. Behroozi, "Light speed reduction to 17 metres per second in an ultracold atomic gas," Nature (London) 397, 594-598 (1999).

6. M. O. Scully and M. S. Zubairy, Quantum Optics (Cambridge U. Press, Cambridge, UK, 1997).

7. M. D. Lukin and A. Imamoglu, "Controlling photons using electromagnetically induced transparency," Nature (London) 413, 273-276 (2001).

8. E. Yablonovitch, "Inhibited spontaneous emission in solidstate physics and electronics," Phys. Rev. Lett. 58, 20592062 (1987)

9. J. Sajeev, "Strong localization of photons in certain disordered dielectric superlattices," Phys. Rev. Lett. 58, 2486 2489 (1987)

10. J. D. Joannopoulos, R. D. Meade, and J. N. Winn, Photonic Crystals: Molding the Flow of Light (Princeton U. Press, Princeton, N.J., 1995).

11. M. Notomi, K. Yamada, A. Shinya, J. Takahashi, C. Takahashi, and I. Yokohama, "Extremely large group-velocity dispersion of line-defect waveguides in photonic crystal slabs," Phys. Rev. Lett. 87, 235902(1-4) (2001).

12. A. Yariv, Y. Xu, R. K. Lee, and A. Scherer, "Coupledresonator optical waveguide: a proposal and analysis," Opt. Lett. 24, 711-713 (1999).

13. S. Mookherjea and A. Yariv, "Second-harmonic generation with pulses in a coupled-resonator optical waveguide," Phys. Rev. E 65, 026607(1-8) (2002).
14. S. Mookherjea and A. Yariv, "Coupled resonator optical waveguides," IEEE J. Sel. Top. Quantum Electron. 8, 448456 (2002).

15. M. Bayindir, B. Temelkuran, and E. Ozbay, "Tight-binding description of the coupled defect modes in threedimensional photonic crystals," Phys. Rev. Lett. 84, 21402143 (2000).

16. See, for example, N. W. Ashcroft and N. D. Mermin, SolidState Physics (Saunders, Philadelphia, Pa., 1976).

17. E. Lidorikis, M. M. Sigalas, E. N. Economou, and C. M. Soukoulis, "Tight-binding parameterization for photonic band gap materials," Phys. Rev. Lett. 81, 1405-1408 (1998).

18. S. G. Johnson and J. D. Joannopoulos, "Block-iterative frequency-domain methods for Maxwell's equations in a plane-wave basis," Opt. Express 8, 173-190 (2001).

19. For a review, see A. Taflove, Computational Electrodynamics: The Finite-Difference Time-Domain Method (Artech, Norwood, Mass., 1995).

20. J. P. Berenger, "A perfectly matched layer for the absorption of electromagnetic waves," J. Comput. Phys. 114, 185-200 (1994).

21. A. Mekis, J. C. Chen, I. Kurland, S. Fan, P. R. Villeneuve, and J. D. Joannopoulos, "High transmission through sharp bends in photonic crystal waveguides," Phys. Rev. Lett. 77, 3787-3790 (1996)

22. S. Fan, S. G. Johnson, J. D. Joannopoulos, C. Manolatou, and H. A. Haus, "Waveguide branches in photonic crystals," J. Opt. Soc. Am. B 18, 960-963 (1998).

23. In accord with the frequency-domain prediction, the active region therefore needs to be approximately 16 coupled cavities long if $\delta n / n_{H}=0.002$; we find that we actually need $\delta n / n_{H}=0.00166$ in the time-domain calculation. We at tribute most of the discrepancy to the inadequacy of the tight-binding approximation, the finite bandwidth of the beam, and the numerical inaccuracies of the simulations.

24. One might wonder about the practicality of applying a large positive field in one arm and a large negative field in the other arm of such a tiny device. All that is required, however, is to establish a strong gradient of the field between the two arms.

25. S. Fan, P. R. Villeneuve, J. D. Joannopoulos, and H. A Haus, "Channel drop tunneling through localized states," Phys. Rev. Lett. 80, 960-963 (1998).

26. S. G. Johnson and J. D. Joannopoulos, "Threedimensionally periodic dielectric layered structure with omnidirectional photonic band gap," Appl. Phys. Lett. 77, 3490-3492 (2000)

27. M. L. Povinelli, S. G. Johnson, S. Fan, and J. D. Joannopoulos, "Emulation of two-dimensional photonic crystal defect modes in a photonic crystal with a three-dimensional photonic band gap," Phys. Rev. B 64, 0753131(1-8) (2001). 